\title{
A Comparison of Leaf Area Index Maps Derived from Multi-Sensor Optical Data Acquired over Agricultural Areas
}

\author{
Giuseppe Satalino*1, Francesco Mattia ${ }^{1}$, Anna Balenzano ${ }^{1}$, Michele Rinaldi², \\ Sergio Ruggieri², Pasquale Garofalo ${ }^{2}$ \\ ${ }^{1} I S S I A-C N R$ - Istituto di Studi sui Sistemi Intelligenti per l'Automazione \\ Via Amendola 122 D-I, 70126 Bari, Italy \\ ${ }^{2}$ CRA - Unità di Ricerca per $i$ Sistemi Colturali degli Ambienti caldo-aridi, \\ Via Celso Ulpiani 5, 70125 Bari, Italy
}

Received: 29 July 2009. Accepted: 10 December 2009.

\begin{abstract}
The objective of this study is to retrieve and compare Leaf Area Index (LAI) maps from temporal series of SPOT, IKONOS and MERIS images acquired, from 2006 to 2008, over an agricultural site in Southern Italy. Results show that the root mean square error (RMSE) of LAI derived from MERIS data is approximately $1 \mathrm{~m}^{2} \mathrm{~m}^{-2}$, slightly larger than the one obtained by using SPOT and IKONOS data. In addition, LAI retrieved from MERIS data tends to underestimate LAI retrieved from SPOT and IKONOS data, particularly at low LAI values. Nevertheless, the paper gives examples highlighting the strength of MERIS with respect to SPOT and IKONOS data in providing long and dense temporal series of LAI maps suitable to feature the temporal evolution of vegetation growth at regional scale.
\end{abstract}

Key-words: LAI, multi-temporal classification, optical data, spatial resolution, SPOT, IKONOS, MERIS

\section{Introduction}

Leaf area index is one of the most important driving variables for crop growth models, as it is often used for their calibration leading to substantial improvements in the model predictions (Jongschaap, 2006). In this respect, the possibility of obtaining spatial and temporal distribution of LAI estimates from remote sensing data is appealing to improve, for instance, yield forecast or water stress prediction over large areas (Dente et al., 2008; Pauwels et al., 2007; Launay and Guerif, 2005). The LAI retrieval from visible and near-infrared (VIS-NIR) remote sensed data is principally based on three alternative approaches, namely the relationships of LAI with vegetation indexes, the inversion of canopy reflectance models and the use of hybrid or semi-empirical models (Guissard et al.,
2005). The accuracy of retrieved LAI is related to several factors, including the sensor spectral characteristics, its spatial resolution and the adopted algorithm. Currently, the availability of optical data acquired with a broad range of temporal and spatial resolutions offers various possibilities to derive LAI maps. For instance, SPOT images (pixel resolution of $10 \mathrm{~m}$, with a swath width of $60 \mathrm{Km}$ for SPOT5) allow accurate LAI retrieval at high spatial resolution, i.e. at field scale (Clevers et al., 2002), whereas IKONOS images (pixel resolution of $4 \mathrm{~m}$, with a swath width of $13 \mathrm{Km}$ ) can provide LAI estimates at very high spatial resolution, i.e. at the sub-field scale suited for precision farming applications (Colombo et al., 2003). However, these sensors are not designed to acquire systematically temporal series of images at global 
scale. In fact, they work on demand and the frequency of their acquisitions changes all over the world (Wulder et al., 2008).

On the other hand, MERIS data are systematically acquired with a high revisiting time and at global scale (i.e. global coverage of the Earth in 3 days with a swath width of $1150 \mathrm{~km}$ ), although at the coarser resolution of $300 \mathrm{~m}$. In this respect, MERIS sensor can deliver dense temporal series of LAI maps that can be integrated into crop growth models for improving the agricultural and water management at global scale (Dash and Curran, 2007). However, to date, relatively little work has been done to assess the accuracy of LAI maps derived from data gathered using MERIS over agricultural sites, notwithstanding it is crucial for their appropriate assimilation into crop growth models.

In this context, the objective of this work is to assess the accuracy of LAI maps derived from MERIS data through a comparison with maps obtained from SPOT and IKONOS data, which have been validated by means of in situ measurements. The exploited data set consists of a three-year temporal series of ground and remote sensing data acquired over an agricultural area located on the Capitanata plain, Southern Italy.

In the next section, the data set is described, then the data analysis and the LAI mapping methods are illustrated, and finally, results are discussed.

\section{Materials and methods}

The site chosen for the experiment is an agricultural area of approximately $700 \mathrm{Km}^{2}$ located in the Capitanata plain, close to the town of Foggia in the Puglia region, Southern Italy. The area has a flat topography and is mainly dedicated to wheat cultivation. According to the local crop management scheduling, durum wheat is usually sown between November and the end of December and harvested at mid June. Other annual main crops of the region are: sugar beet (sown in autumn and harvested in July), and tomato (sown in April and harvested in AugustSeptember). Permanent crops with a significant presence in the area are vineyards and olives. Figure 1 shows the experimental area and an updated land cover map. In the following subsections, ground and optical data are presented.

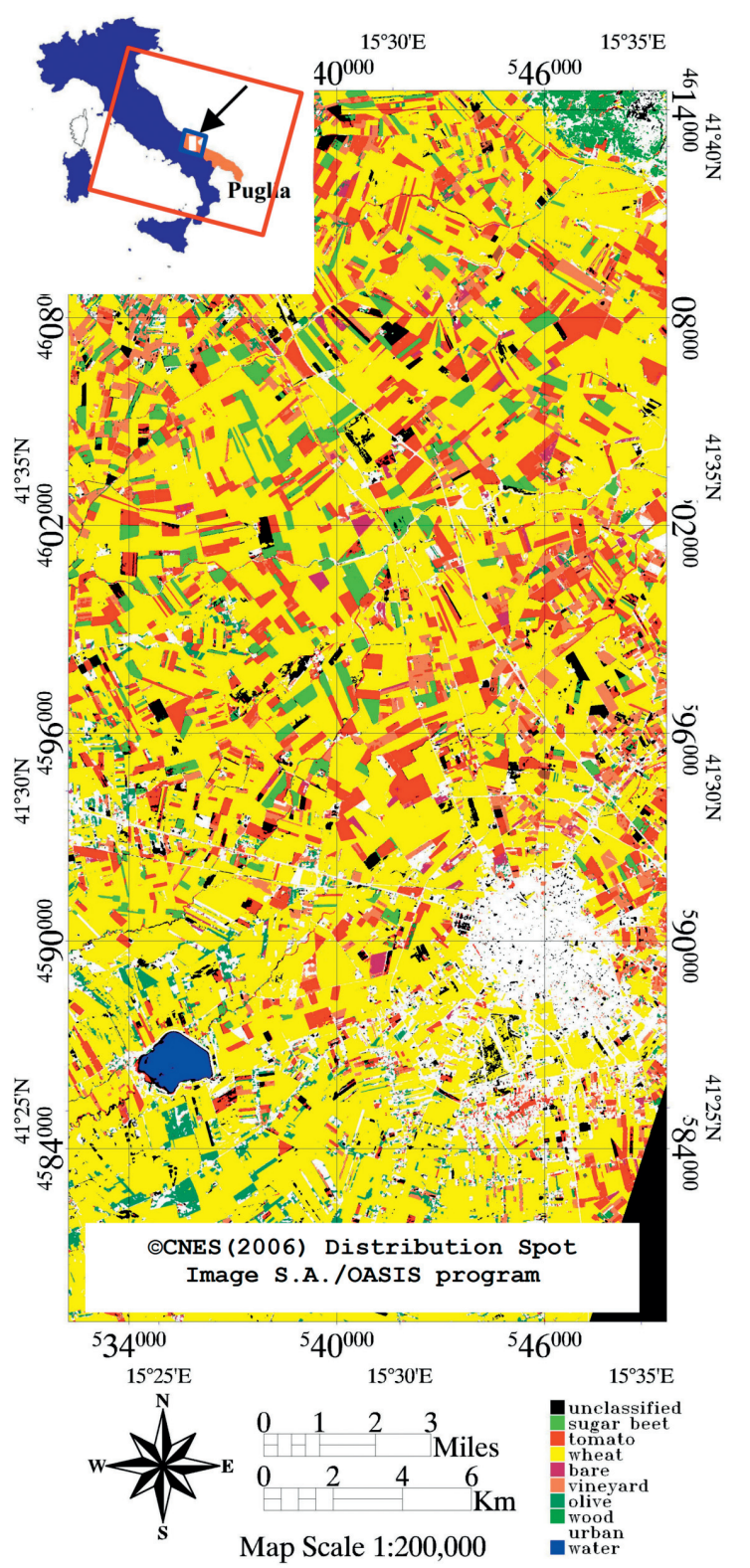

Figure 1. The experimental area in the Puglia region (shown by the white rectangle) and the classified image obtained from multi-temporal SPOT data in 2007. Swaths of MERIS (in red) and SPOT (in blue) images are superimposed. The swath of IKONOS is contained inside the white rectangle.

\subsection{Ground data}

From 2006 to 2008, ground data were intensively collected over 5 selected experimental farms, in the framework of a national project (Rinaldi et al., 2007, Satalino et al., 2009). In particular, vegetation biomass, soil moisture content at various depths, soil temperature, and LAI measure- 
Table 1. Number of fields monitored during the measurement campaigns, and number of fields surveyed during the days of the additional measurements.

\begin{tabular}{lccc}
\hline CROP & 2006 & 2007 & 2008 \\
\hline Monitored: Wheat & 4 & 5 & 7 \\
Monitored: Beet & 5 & 3 & 3 \\
Monitored: Tomato & 3 & 4 & 4 \\
Surveyed: Wheat & - & - & 11 \\
Surveyed: Beet & - & 10 & 14 \\
Surveyed: Tomato & - & 37 & 23 \\
\hline
\end{tabular}

ments (by means of the LiCOR LAI-2000 instrument) were acquired over wheat, beet and tomato fields. The number of fields monitored during the growing seasons is reported in Table 1 , and for each field and each date, 6 LAI measurements were collected and subsequently averaged at field scale. For example, Figure 2 shows the temporal behaviour of LAI observed over a field of wheat, sugar beet and tomato. The error bars are equal to the standard deviation of the in situ measurements per each date. In addition, the plot reports the temporal trend obtained by interpolating the observed data with a polynomial of second degree. It is worth mentioning that for each year, there is an overlap between the growth curves of the three crops. On the other hand, between subsequent years, a temporal shift of the growth curves has also been observed. Such a shift is mainly due to the change in the sowing dates and to the inter-annual climate differences.

Part of the LAI measurements were collected during 5 days of additional intensive measurements (i.e. on DoY 187 and 215, 2007; on DoY 120, 172 and 191,2008) and they have been used as an independent data set to validate NDVI-LAI relationships for SPOT and IKONOS data.

\subsection{Optical data}

During the same growing seasons, SPOT, IKONOS and MERIS data were acquired over the Capitanata plain. The SPOT images have pixel resolutions ranging between $10 \mathrm{~m}$ and 20 $\mathrm{m}$ (because they were actually acquired by a number of various SPOT sensors, i.e. SPOT5, SPOT4 or SPOT2), whereas the IKONOS and MERIS (full resolution product, level 1P) data have a pixel resolution of $4 \mathrm{~m}$ and $300 \mathrm{~m}$, respectively. The dates of the gathered images are reported in Table 2, while the swaths of MERIS, SPOT and IKONOS systems over the study area are shown in Figure 1 . The dates of MERIS acquisitions coincide or are close to those of both SPOT and IKONOS. Whereas, due to conflicts with higher priority acquisitions, it has not been possible to obtain simultaneous acquisitions of SPOT and IKONOS images (indeed they have temporal shifts longer than 9 days). It may be worth mentioning that the list of MERIS data shown in Table 2 is a subset of all MERIS data acquired over the area. All the images have been projected in the same map projection and the geo-location accuracy has been checked to be of approximately 1 pixel.

The multi-temporal SPOT images acquired over the area have also been employed to derive updated and accurate land cover maps of the area under investigation for 2006, 2007 and 2008. For example, the land cover map obtained from SPOT data acquired in 2007 is shown in Figure 1 . The map has been obtained by using the maximum likelihood algorithm. In 2007, the main classes and their coverage percentages in the study area were: wheat $(54 \%)$, sugar beet $(3 \%)$, tomato $(10 \%)$, vineyard $(7 \%)$, and olive (5\%). In the other two years (2006 and 2008), the coverage percentages obtained for the nonpermanent crops (i.e. wheat, sugar beet and tomato) were approximately the same, although some fields were sown with different crops due to the crop rotation practice.

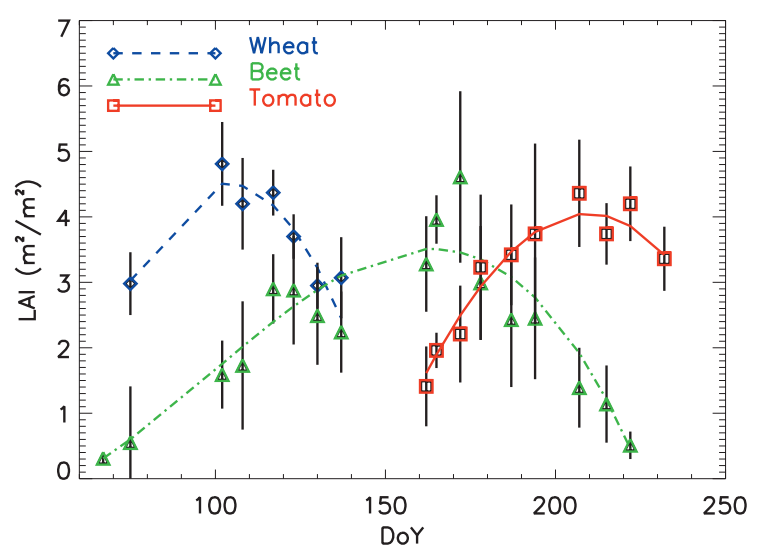

Figure 2. LAI temporal behaviours for a field of wheat, sugar beet and tomato in 2007. 
Table 2. Pairs of SPOT-MERIS and IKONOS-MERIS data acquired during the measurement campaigns.

\begin{tabular}{lcccc}
\hline & SPOT & & \multicolumn{2}{c}{ MERIS } \\
\hline DoY & Date & Type & DoY & Date \\
\hline 146 & 20060526 & SPOT5 & 142 & 20060522 \\
182 & 20060701 & SPOT4 & 180 & 20060629 \\
212 & 20060731 & SPOT5 & 212 & 20060731 \\
113 & 20070423 & SPOT5 & 114 & 20070424 \\
171 & 20070620 & SPOT5 & 174 & 20070623 \\
228 & 20070816 & SPOT5 & 229 & 20070817 \\
104 & 20080413 & SPOT4 & 111 & 20080420 \\
199 & 20080717 & SPOT2 & 198 & 20080716 \\
\hline & IKONOS & & \multicolumn{2}{c}{ MERIS } \\
\hline DoY & Date & & DoY & Date \\
\hline 187 & 20070706 & & 187 & 20070706 \\
215 & 20070803 & & 219 & 20070807 \\
130 & 20080509 & & 124 & 20080503 \\
176 & 20080624 & & 178 & 20080626 \\
190 & 20080708 & & 185 & 20080703 \\
\hline
\end{tabular}

\subsection{LAI mapping methods}

The methodology to derive LAI maps depends primarily on the optical sensor, as illustrated in the following sub-sections.

\subsubsection{LAI retrieval from SPOT and IKONOS} data. LAI maps derived from SPOT and IKONOS data have been obtained by means of empirical relationships based on the Normalized Difference Vegetation Index (NDVI). The use of multi-temporal data can introduce errors due to differences of sun illumination, viewing geometry, etc. To reduce the effects of these factors on the NDVI calculation, SPOT and IKONOS bands have been normalised converting the Digital Number (DN) into the Top Of Atmosphere (TOA) reflectance. Since the experimental area is fairly flat, the effects of the local topography have been disregarded. Moreover, the atmospheric conditions during the SPOT and IKONOS acquisitions were good; therefore no atmospheric corrections have been applied.

Three previously proposed fit functions relating LAI to NDVI (i.e. linear, exponential, and logarithmic functions (Qi et al., 2000)) have been assessed. The parameters of the fit functions have been computed by means of a statistical fit on pairs of NDVI-LAI values computed at field scale. To obtain the temporal matching between SPOT or IKONOS derived-
NDVI and in situ measured LAI values, the latter have been interpolated.

To improve the accuracy, the fit parameters have been calibrated separately for each crop of interest (Colombo et al., 2003). After the calibration, LAI maps for each crop can be obtained by using an updated land-cover map. The strength of this method is that it can be tuned to specific conditions and sites, whereas its weakness is that it requires a considerable number of ground measurements and that it cannot be easily extrapolated to other sites and/or crops.

\subsubsection{LAI retrieval from MERIS data. LAI maps} derived from MERIS data were obtained by means of a hybrid algorithm, referred to as TOA_VEG (Baret et al., 2006), available in the BEAM software (Brockmann, 2008). The algorithm estimates LAI by means of a Neural Network (NN) that inverts MERIS reflectance. NN is trained on the basis of MERIS simulated data obtained by the SAIL (Scattering by Arbitrarily inclined Leaves) model for the canopy reflectance, coupled to the PROSPECT model for estimating leaf-level reflectance and transmittance, and by the SMAC (Simplified Method for the Atmospheric Correction) model used for the top of atmosphere reflectance. This algorithm is not designed for specific crops. Therefore, its strength is that it can be applied to various types of vegetation, whereas its weakness is that it can be less accurate.

\section{Results}

The assessment of the obtained results has been performed in four steps: (a) calibration of SPOT and IKONOS NDVI-LAI fit functions by using LAI in-situ measurements; (b) validation at field scale of the obtained LAI maps by using independent LAI measurements; (c) retrieval of SPOT and IKONOS LAI estimates at field scale by using updated land-cover maps and field boundaries available in a GIS format; (d) comparison of field-averaged LAI estimates derived from MERIS with those derived from SPOT and IKONOS. To reduce the effects of the mixed pixels, the latter comparison has been carried out only over fields larger than 25 hectares. 


\subsection{LAI retrieved from SPOT data}

The fit functions have been calibrated for each crop by using three-year NDVI-LAI series of data collected over the monitored fields listed in Table 1. Data refers to NDVI extracted from SPOT acquired on the dates listed in Table 2. The overall number of data pairs is 16, 22 and 18 for wheat, beet, and tomato, respectively. The fit functions considered are linear, exponential, and logarithmic. The accuracies in terms of root mean square error (RMSE) between LAI measured (or interpolated from the growth curves) and LAI retrieved from NDVI by using the linear function are $0.76,0.90$ and $0.79 \mathrm{~m}^{2} \mathrm{~m}^{-2}$ for wheat, sugar beet and tomato, respectively. The accuracies obtained by using the other two functions are very similar, therefore the linear function has been selected. The bias and slope coefficients of the NDVI-LAI linear functions are: $(1.99,3.15)$ for wheat, $(-0.43,5.46)$ for sugar beet, and $(-2.22,12.42)$ for tomato. As a general comment, results show that RMSE values for the three crops are comparable, although slightly higher for sugar beet. Figure 3 illustrates the scatter plot of LAI measured versus LAI retrieved from SPOT data by using the NDVILAI linear function. The scatter plot shows that LAI retrieved is not biased and that its slope is close to 1 . An example of a LAI map obtained from SPOT data acquired on April 13 ${ }^{\text {th }}, 2008$ (DoY 104), is reported in Figure 4. The poligons on the map refer to wheat or sugar beet fields at different phenological stages. Poligons related to tomato fields are not shown because the tomato crop was not yet grown.

As an additional step, the LAI values retrieved from SPOT data have been assessed versus independent in situ measurements collected during the additional measurements carried out in 2007 and 2008. More precisely, LAI measurements on DoY 187, 2007 and on DoY 191, 2008 have been selected to be compared with LAI values retrieved from SPOT images acquired on DoY 171, 2007 (i.e. 16 days earlier than the closest in situ measurements) and on DoY 199, 2008 (i.e. 8 days later than the closest in situ measurements). As a result, 9 and 33 NDVI-LAI data pairs have been obtained for sugar beet and tomato, respectively. Since the above mentioned temporal shifts occurred during critical stages of the crop growing periods

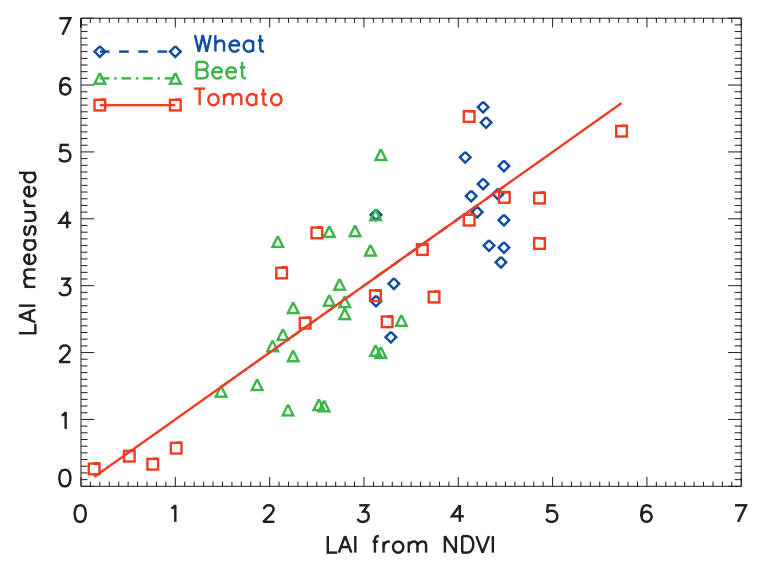

Figure 3. Scatter plot of LAI measured versus LAI retrieved from SPOT data by using the NDVI-LAI linear function.

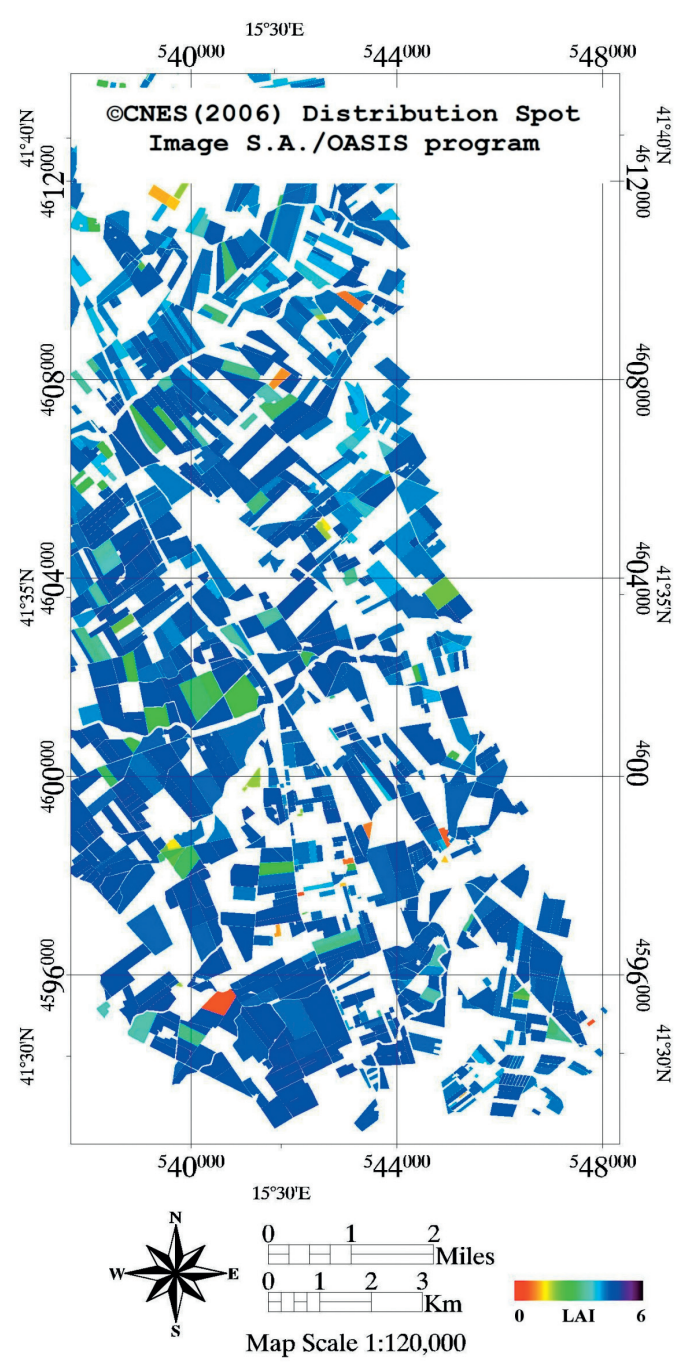

Figure 4. LAI map obtained from SPOT data acquired on April $13^{\text {th }}$, 2008. Poligons refer to wheat and sugar beet fields at different phenological stages. 
(see Figure 2), larger discrepancies than those previously reported are expected. Indeed, the overall RMSE between LAI-retrieved and LAImeasured is approximately $1.46 \mathrm{~m}^{2} \mathrm{~m}^{-2}$ for sugar beet and $1.45 \mathrm{~m}^{2} \mathrm{~m}^{-2}$ for tomato. Unfortunately, for wheat, no data is available for the validation.

\subsection{LAI retrieved from IKONOS data}

Following a similar scheme, NDVI-LAI linear functions have been firstly calibrated and then, LAI retrieved from IKONOS has been assessed versus independent in situ measurements. The LAI measurements used to calibrate the LAI fit functions were collected in 2008, close or coincident to the IKONOS acquisitions. The RMSE between LAI measured and retrieved is 0.88 , 0.88 , and $1.01 \mathrm{~m}^{2} \mathrm{~m}^{-2}$ for wheat, sugar beet, and tomato, respectively. Whereas, the bias and slope of the NDVI-LAI linear functions are: $(-2.14,10.34)$ for wheat, $(-0.42,6.52)$ for sugar beet and $(-0.13,6.19)$ for tomato. Also in this case, results show that RMSE values for the three crops are comparable, although slightly higher for tomato. Figure 5 illustrates the scatter plot of LAI measured versus LAI retrieved from IKONOS data. It shows that LAI retrieved is not biased and its slope is close to 1 . The LAI accuracies derived from IKONOS are similar to those derived from SPOT for sugar beet, whereas a slight difference for wheat and tomato has been found. These differences can be due to several factors, such as the number of LAI measurements used, the inter-calibration errors of

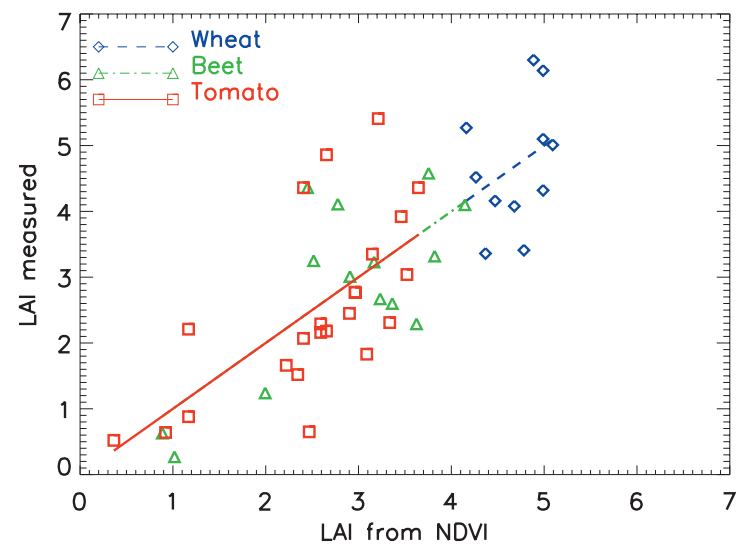

Figure 5. Scatter plot of LAI measured versus LAI retrieved from IKONOS data by using the NDVI-LAI linear function.

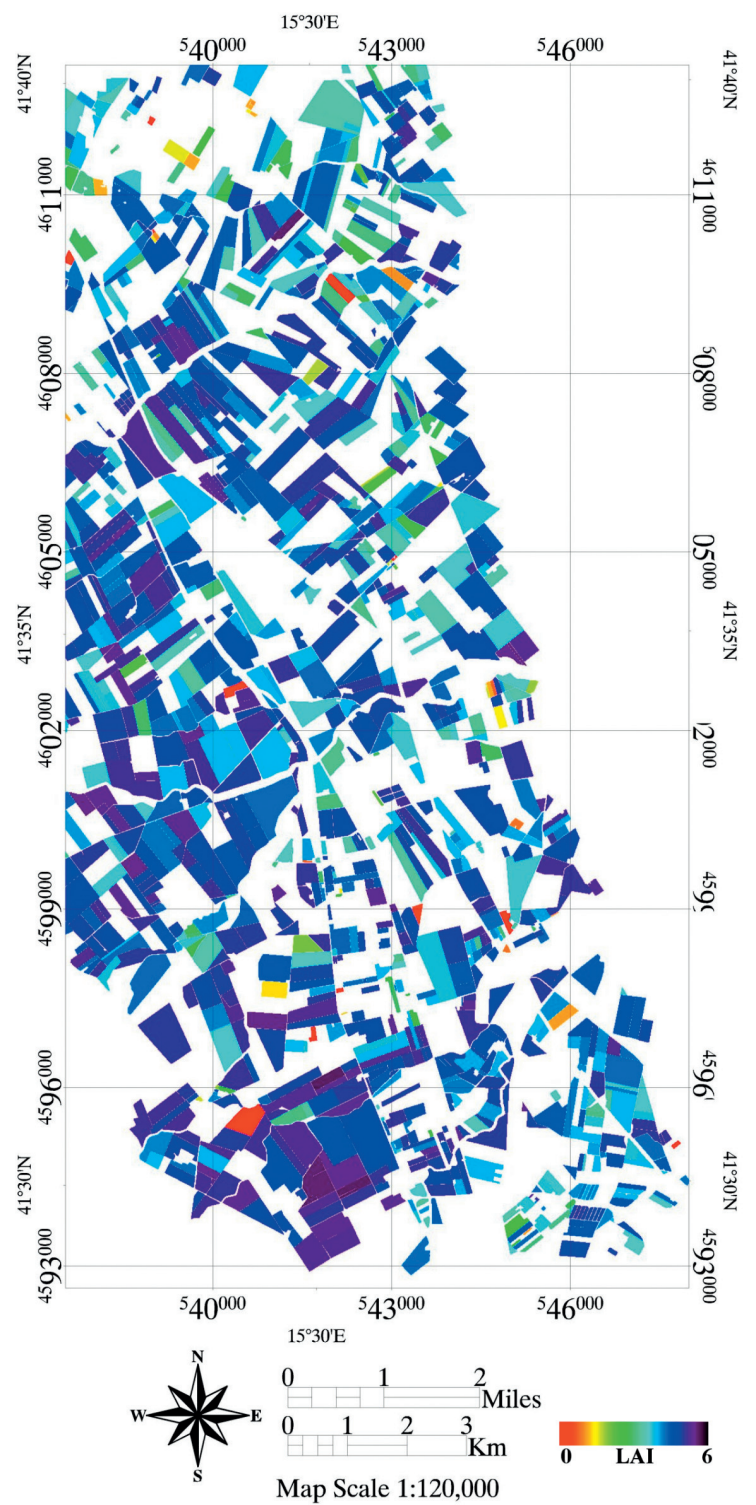

Figure 6. LAI map obtained from IKONOS data acquired on May $9^{\text {th }}, 2008$. Poligons refer to wheat and sugar beet fields at different phenological stages.

the different dates of the SPOT and IKONOS temporal series (Soudani et al., 2006).

An example of LAI map obtained from IKONOS data acquired on May 9 $9^{\text {th }}, 2008$ (DoY 130 ), is reported in Figure 6. The poligons refer to wheat or sugar beet fields at different phenological stages. Between this LAI map and the one derived from SPOT data reported in Figure 4, there is a temporal shift of 26 days. As a consequence, the LAI map estimated on DOY 130 is generally higher for both crops. 
The validation of the NDVI-LAI relationships has been carried out by using the additional LAI measurements acquired in 2007 (Table 2). The RMSE between LAI retrieved from NDVI and LAI measured is 0.48 and 1.17 $\mathrm{m}^{2} \mathrm{~m}^{-2}$ for sugar beet and tomato, respectively. The higher error obtained for tomato is probably due to the fact that tomato is a crop with a complex structure not completely covering the soil. For wheat, no data is available for the validation.

\subsection{LAI retrieved from MERIS data}

The temporal series of LAI maps derived from MERIS images listed in Table 2 have been obtained by using the TOA-VEG algorithm. The expected RMSE for LAI estimates reported in literature is approximately $1 \mathrm{~m}^{2} \mathrm{~m}^{-2}$ for LAI values up to $4 \mathrm{~m}^{2} \mathrm{~m}^{-2}$. To improve the comparison between these LAI maps with those derived from SPOT and IKONOS, fields with an extension larger than 25 hectares have been selected.

\subsection{MERIS and SPOT LAI maps comparisons}

LAI maps derived from MERIS and SPOT have been compared over 93 fields of wheat and 22 fields of sugar beet. RMSE values obtained comparing field-averaged LAI derived from MERIS and from SPOT are $1.04 \mathrm{~m}^{2} \mathrm{~m}^{-2}$ for wheat fields and $1.01 \mathrm{~m}^{2} \mathrm{~m}^{-2}$ for sugar beet fields, whereas the fit lines have bias and slope equal to $(-3.33,1.61)$ and $(-0.64,1.02)$, respectively. Figure 7 shows LAI retrieved from MERIS versus LAI retrieved from SPOT for the two crops. In general, LAI retrieved from MERIS underestimates LAI retrieved from SPOT, in particular when LAI is low. In addition, RMSE obtained for LAI retrieved over tomato fields is higher than RMSE obtained for wheat and sugar beet crops. The reasons are probably due to image co-registration errors and to the coarse pixel resolution of MERIS data that renders unavoidable the presence of mixed pixels. In addition, in the case of tomato crop, the algorithm under-performance can be again related to the fact that the canopy does not cover the soil completely. Indeed, this effect needs further investigations.

\subsection{MERIS and IKONOS LAI maps comparisons}

LAI maps derived from MERIS and IKONOS have been compared over 33 fields of wheat and 9 fields of sugar beet. RMSE values are $1.07 \mathrm{~m}^{2}$

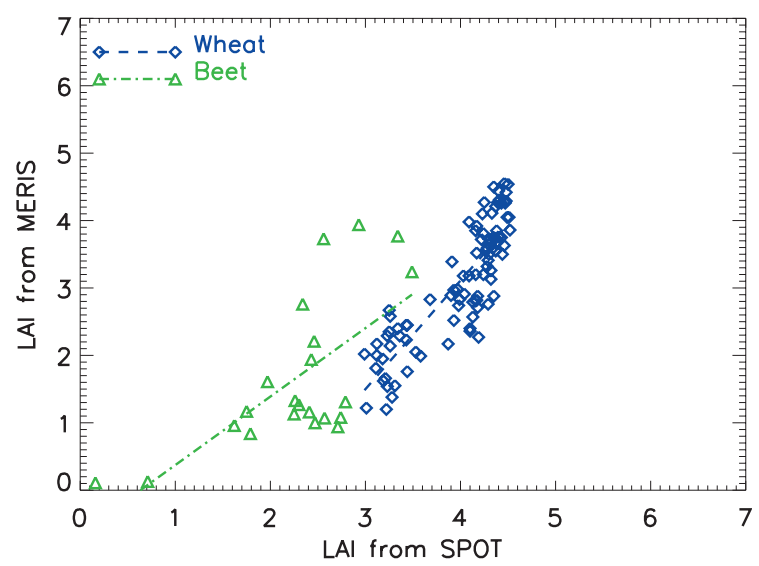

Figure 7. Scatter plot of LAI retrieved from MERIS versus LAI retrieved from SPOT.

$\mathrm{m}^{-2}$ for wheat fields and $0.99 \mathrm{~m}^{2} \mathrm{~m}^{-2}$ for sugar beet fields, whereas the scatter plots have bias and slope equal to $(0.88,0.59)$ and $(-0.45,0.89)$, respectively. Figure 8 shows LAI retrieved from MERIS versus LAI retrieved from IKONOS for the two crops. Again, LAI retrieved from MERIS underestimates the LAI retrieved from IKONOS, in particular when LAI is low. For tomato, also in this case, larger LAI estimate errors have been found.

\section{Discussion and conclusions}

In this study, LAI maps derived from SPOT, IKONOS and MERIS data have been compared and their accuracy assessed over wheat, sugar beet and tomato crops located on an agri-

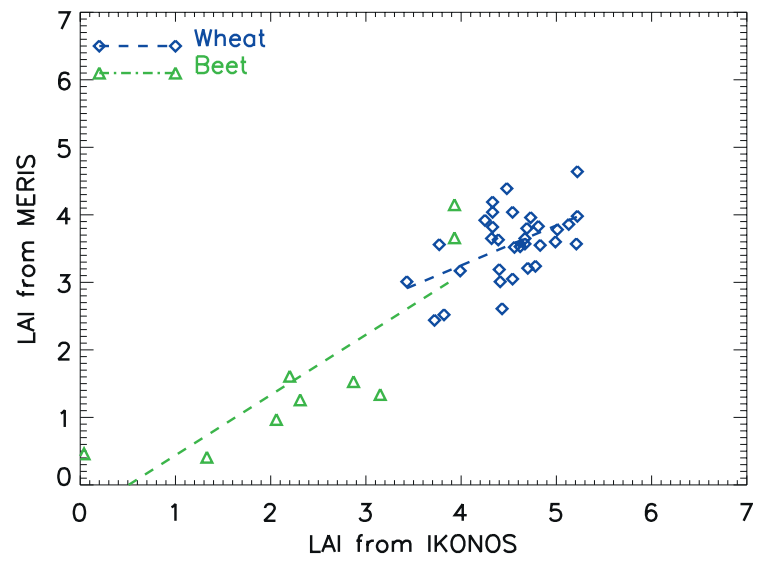

Figure 8. Scatter plot of LAI retrieved from MERIS versus LAI retrieved from IKONOS. 
cultural site in Southern Italy. Temporal series of ground and SPOT, IKONOS and MERIS data acquired during the 2006-2008 growing seasons have been analyzed. Firstly, NDVI-LAI linear regression functions have been derived by using SPOT and IKONOS NDVI data and LAI in situ measurements. Then, the obtained relationships have been validated by using an independent experimental data set. Subsequently, LAI derived from MERIS have been compared with LAI derived from SPOT and IKONOS over large fields in order to reduce the effect of the mixed pixels. The RMSE between LAI derived from MERIS and SPOT is approximately $1.04 \mathrm{~m}^{2} \mathrm{~m}^{-2}$ for wheat fields, and $1.01 \mathrm{~m}^{2} \mathrm{~m}^{-2}$ for sugar beet fields. Whereas, the RMSE between LAI derived from MERIS and IKONOS is approximately $1.07 \mathrm{~m}^{2} \mathrm{~m}^{-2}$ for wheat fields, and $0.99 \mathrm{~m}^{2} \mathrm{~m}^{-2}$ for sugar beet fields. In general, results show that LAI retrieved from MERIS slightly underestimates the LAI retrieved from SPOT and IKONOS images, especially for low LAI values. For tomato fields, errors are higher, and these observations require further investigations.

These results show that LAI maps derived from high and very high resolution remote sensing data (i.e. SPOT and IKONOS) are accurate and their accuracy can be improved by using well-calibrated multi-temporal images, and specific NDVI-LAI functions. As a result, the obtained maps can reveal not only inter-field but also intra-field LAI differences, useful in precision farming.

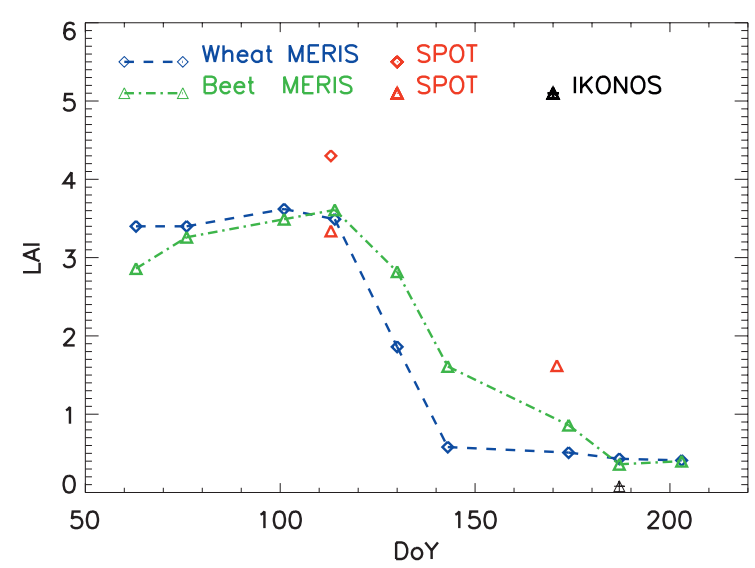

Figure 9. Temporal behaviours of LAI estimated in 2007 from MERIS, SPOT and IKONOS data, for a selected wide field of wheat and sugar beet.
On the other hand, LAI maps derived from MERIS data, despite they have a coarser resolution that does not permit the identification of small fields, still have acceptable accuracy (i.e. approximately $1.0 \mathrm{~m}^{2} \mathrm{~m}^{-2}$ ). Nevertheless, the comparisons show that LAI retrieved from MERIS data underestimates LAI retrieved from SPOT or IKONOS, in particular at low LAI values.

In this respect, the strength of MERIS data is that they are systematically acquired with a short revisiting time offering the possibility of collecting long and dense temporal series of LAI maps. For example, Figure 9 shows the temporal behaviour of LAI-MERIS estimated in 2007 for two selected large fields of wheat and sugar beet. The temporal series of MERIS data consists of 9 dates that clearly show the LAI evolution of the two crops. In contrast, in the same period there are only few LAI values obtained from SPOT and IKONOS, not enough to infer the LAI temporal evolution for the two crops. As a further example, Figure 10 shows a synoptic view of LAI temporal evolution in 2007 over the Capitanata plain. The figure shows a RGB temporal composition of three LAI maps obtained from MERIS data acquired

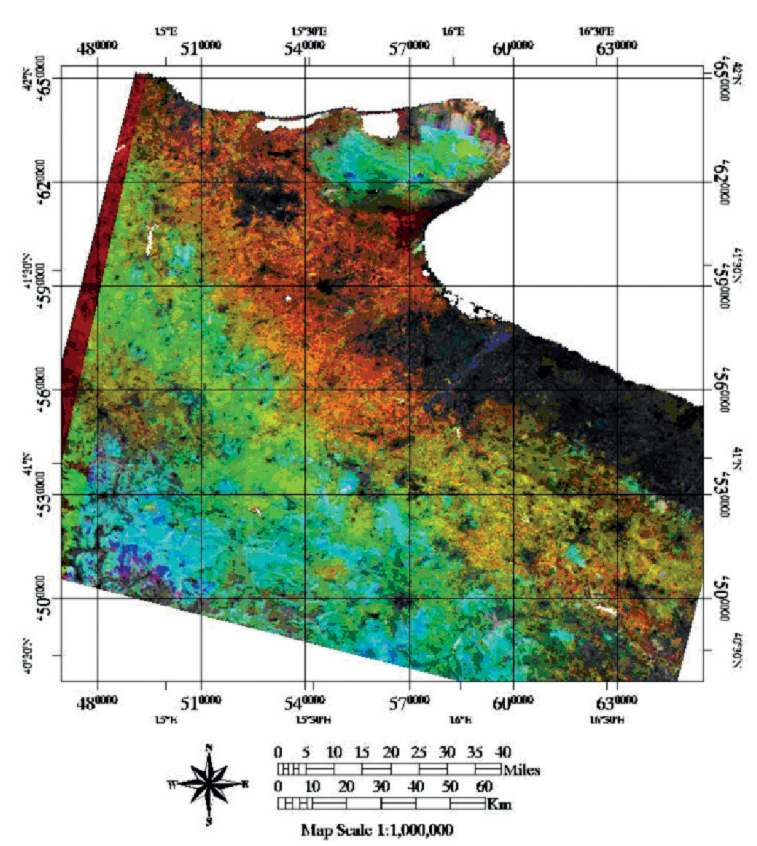

Figure 10. Multi-temporal LAI map obtained from MERIS on 2007 over the Capitanata plain. The colours were obtained by associating LAI maps on dates $(11.04 .07,10.05 .07,30.06 .07)$ to $(\mathrm{R}, \mathrm{G}, \mathrm{B})$ channels. 
in 2007 (LAI maps referring to April, 11; May, 5 and June, 7 are associated to R, G and B colours, respectively). The different colour patterns show the geographical dispersion of the various crops present in the agricultural area. For example, the reddish colour means that the red and green channels have higher values (i.e. LAI in April and May higher than LAI at the end of June) and this occurs in areas where wheat and sugar beet are the main crops, as detailed in the updated land cover map shown in Figure 1.

\section{Acknowledgments}

This work was partly supported by the Italian Ministry of Agriculture, Food and Forestry Policies (AQUATER project, contract nr. 209/7303/05) and partly by ESA-ESTEC (ELASIM project, contract nr. 19558/06/NL/HE). SPOT data were supplied from CNES (2006/2009) Distribution Spot Image S.A./OASIS/ISIS program, and MERIS data within the ENVISAT AO 662 and ESA AOALO 3597.

\section{References}

Baret F., Pavageau K., Beal D., Weiss M., Berthelot V., Regner P. 2006. Algorithm Theoretical Basis Document for MERIS Top of Atmosphere Land Products (TOA_VEG). Mar. 2006, INRA-Novelties.

Brockmann C. 2008. BEAM software, http://www. brockmann-consult.de/beam.

Clevers J.G.P.W., Vonder O.W., Jongschaap R.E.E., Desprats J.F., King C., Prevot L., Bruguier N. 2002. Using SPOT data for calibrating a wheat growth model under mediterranean conditions. Agronomie, 22:687-694.

Colombo R., Bellingeri D., Fasolini D., Marino C.M. 2003. Retrieval of leaf area index in different vegetation types using high resolution satellite data. Remote Sens. Environ., 86:120-131.

Dash J., Curran J. 2007. Relationship between the MERIS vegetation indices and crop yield for the state of South Dakota, USA. Proc. Envisat Symposium 2007, Montreux, Switzerland, 23-27 April 2007 (ESA SP-636, July 2007).
Dente L., Satalino G., Mattia F., Rinaldi M. 2008. Assimilation of ASAR and MERIS data into CERES-Wheat model to estimate yield maps. Remote Sens. Environ., 112, 1395-1407, doi:10.1016/j.rse.2007.05.023.

Guissard V., Lucau-Danila C., Defourny P. 2005. Crop specific LAI retrieval using optical and radar satellite data for regional crop growth monitoring and modelling. Proc. SPIE, vol. 5976.

Jongschaap R.E.E., 2006. Run-time calibration of simulation models by integrating remote sensing estimates of leaf area index and canopy nitrogen. Eur. J. Agron., 24:316-324.

Launay M., Guerif M. 2005. Assimilating remote sensing data into a crop model to improve predictive performance for spatial applications. Agriculture, Ecosystems and Environment, 111:321-339.

Pauwels V.R.N., Verhoest N.E.C., De Lannoy G.J.M., V. Guissard, C. Lucau, and P. Defourny (2007), Optimization of a coupled hydrology-crop growth model through the assimilation of observed soil moisture and leaf area index values using an ensemble Kalman filter. Water Resour. Res., 43, W04421, doi:10.1029/ 2006WR004942.

Qi J., Kerr Y.H., Moran M.S., Weltz M., Huete A.R., Sorooshian S., Bryant R. 2000. Leaf Area Index Estimates Using Remotely Sensed Data and BRDF Models in a Semiarid Region. Remote Sens. Environ., 73:18-30.

Soudani K., François C., le Maire G., Le Dantec V., Dufrêne E. 2006. Comparative analysis of IKONOS, SPOT, and ETM+ data for leaf area index estimation in temperate coniferous and deciduous forest stands. Remote Sens. Environ., 102:161-175.

Rinaldi M., Campi P., Castellini M., Ferrara R.M., Fiorentino C., Garofalo P., Satalino G. 2007. AQUATER Project: the Measurement Campaign in Capitanata Plain of Soil-Plant-Atmosphere Continuum. Proc. of the $5^{\text {th }}$ Int. Symp. on Retrieval of Bioand Geophysical Parameters from SAR Data for Land Applications, Bari, Italy, Sept. 25-28.

Satalino G., Mattia F., Le Toan T., Rinaldi M. 2009. Wheat Crop Mapping by using ASAR AP data. IEEE Trans. on Geoscience and Remote Sensing, 47, 2:527-530.

Wulder M.A., White J.C., Goward S.N., Masek J.G., Irons J.R., Herold M., Cohen W.B., Loveland T.R., Woodcock C.E. 2008. Landsat continuity: Issues and opportunities for land cover monitoring. Remote Sens. Environ., 112:955-969. 